\title{
Application of Interaction Design on Visual Communication Design
}

\author{
Zhuo Wang \\ Jilin Animation Institute, Changchun, Jilin, 130000
}

Keywords: application research; interaction design; visual communication

\begin{abstract}
In the common concept, visual communication design is static, and along with the development of modern technology and the Internet, the new term "interaction" has been created. With the wide application of interaction design in various fields, visual communication design is no longer confined to the composition and appearance of two-dimensional elements for the purpose of form beauty, but more and more emphasis on the user's experience of reading the work and Emotional design.
\end{abstract}

\section{Introduction}

Relatively speaking, the idea of interactive design is the expansion of visual communication design, which represents the development of visual communication design to a higher level. Many aspects have bid farewell to the past problems, and the overall value is very prominent. In the current stage of development, China is entering a very special period. All work should be carried out in a targeted manner to achieve higher achievements. Therefore, the implementation of the interactive design concept is very necessary [1].

\section{Concept of Interaction Design Concept}

The existence of the interaction design concept means that in the process of information and data transmission, good interaction effects can be achieved, so as to ensure that each piece of information and data is transmitted in a simple manner, rather than a simple communication. For example, many of the leaflet designs at this stage have made a lot of changes, from the simple text narrative to the beautiful design, and then changed to the field. Most of today's flyers include internal staff introductions, avatars, field scenes, contact numbers, background designs, etc. The purpose is to let people see the flyers and want to go to the field or watch them. Only by fully attracting customers, can we better establish cooperation intentions, and then sign the corresponding contracts to achieve common development. From this point of view, the existence of interactive design concepts is an inevitable product of social and cultural development and technological development. It can, to a large extent, improve traditional interactions, avoid false imaginations and suspicions, and promote interactive design concepts. Get more recognition in the implementation process [2].

\section{Significance of Visual Communication Design}

In the development of modern society, the number and types of various products are constantly increasing. It is not an easy task to get the desired results in the future work. There are many influencing factors to deal with. In addition, with the rapid development of the tertiary industry, the service business has been highly recognized by the society and continues to increase in demand. At this time, visual communication design has become the "darling" of various industries, and the various help it can bring will be very prominent. The analysis believes that the meaning of visual communication design is mainly reflected in the following aspects: First, the implementation of the design content can better transform the customer's many subjective ideas into objective reality, thus achieving a good transition of subjective and objective. For the resolution of contradictions in the communication process, it has greatly promoted. Second, visual communication design is not static. 
On the contrary, it will generate more content according to the development of the times and technological changes. For example, design software is constantly innovating. Today's beautiful plans and software are the product of innovations in visual communication design, and have a great impact on residents' production, life, and work. I believe that in the future work, visual communication design can create higher value. In our busy daily life, we are exposed to visual communication design almost every moment. Whether you're seeing street signs on the road, body ads, window posters, and exquisite menus in restaurants, these beautiful ads are always catching our eye. It can be said that our lives are surrounded by a world full of visual communication design. Visual communication design refers to the styling activities in the two-dimensional space using the graphic language as the medium carrier, including the concept and expression, and the purpose is to convey the information or creativity of the visual media. The meaning of visual communication design for us: It promotes life through the design, planning of symbols, forms, and order of information such as culture, commodities, psychology, emotions, etc., facilitating the popular dissemination and acceptance of information, and facilitating the translation and decoding of emotions. The environment is comfortable, beautiful and safe, which improves the efficiency of behavior, develops deep physical potential, and promotes the convenience, speed and effectiveness of communication in life. For example, in Alexander Finning's "The Helper's Menu" series of posters, the author used bread to shape various foods, showing the lives of people at the bottom of society. The whole work does not have a straightforward statement, but the image implies the exchange and interaction with the readers, which leads to the reader's association and thinking.

\section{Application of Interactive Design Concept in Visual Communication Design}

Judging from the current situation, the advertising industry has developed very rapidly and has had a particularly positive effect on the development of various industries and industries in the country. In simple terms, the purpose of advertising design expansion is to better promote products and publicity services so as to urge residents to better understand emerging issues. In the traditional advertising design process, visual communication design is only passively publicized. However, after the effective use of the interactive design concept, the field of advertising design has made breakthrough progress. For example, when we watch a webpage video, we have advertising. Past ads can only be watched, but today's ads can be exempted by VIP users, and they can also click on the content of advertisements to enter the corresponding interface to purchase products, understand products, contact customer service, etc., thus achieving a kind of customer and The interaction effect of advertisements can also collect more data and information at the same time, which ultimately leads to continuous improvement of the level of advertising design. It is no longer subject to strong hate and opposition.

As far as visual communication design is concerned, in the course of carrying out its work, publicity for various products needs to be completed through certain packaging, and at the same time, many consumers also have more demands for packaging. For this reason, we can fully apply the interactive design concept in the packaging field. Before consumers use the product, the first thing that comes into contact with the product is the packaging of the product. Therefore, the level of packaging design directly affects the status and image of the product in the minds of consumers. Interactive packaging that influences consumer behavior can start with people's senses and use a multi-sensory sense of smell, touch, and vision to bombard consumers with a full range of stereoscopic information in order to enhance the competition in the sale of goods and improve the consumer-to-consumer relationship. This shows that the application of interactive design concepts in packaging design can create high value [3].

The cultural undertakings of the modern society have developed rapidly. Many people have paid great attention to the improvement of their own cultural qualities. Therefore, the demand for books has begun to increase substantially. Applying the interactive design concept can promote the improvement of book design and binding. For example, in the design process of children's books, illustration content can be added, and various cards can be added and spliced, and can also be more prominent in the display of three-dimensional drawings. The content of a children's book can be 
fully displayed using different interactive design concepts. This will not only attract consumers, but also achieve value for money, stimulate the sound development of the book market, and promote the overall progress of cultural propaganda.

Visual communication design includes design content such as print advertisement design, book binding design, packaging design, logo design, and corporate image design. With the advancement of science and technology, the rapid development of digital media technology has changed from design tools to design concepts. The connotation and extension of visual communication design has also accelerated with the pace of the times. At the same time, the importance of modern visual communication design in people's lives has become more and more significant. People have gained open imagination and new ways of experiencing things through visual communication design, releasing imagination and creativity. Interaction in visual communication design is easily overlooked by designers. The main reason is that the interaction of visual communication design mainly uses the visual channel. Unlike the website design, the visual and auditory channel synthesis application can produce rich interactive effects. Like multimedia products are integrated into interactive designs by integrating sensory channels such as sight, hearing, and touch. In fact, the interactive design has a wide range of expressions. However, no matter what form of expression, the core of the interaction design is "human". Its original ideal is to provide users with an experience platform, so that they can have a real personal experience and thus obtain accurate information [4]. The interaction in visual communication design is mainly reflected in three aspects: information transmission, emotional communication, and user participation. Below, let's talk about how the concept of interaction is applied to all areas of visual communication design.

In today's traditional advertising field, although the amount of advertising works is still very large but the response received is flat, the main reason is that traditional advertising is still using a one-way notification method to deliver information, and how can it achieve without interaction with consumers? What is the effect of good information dissemination? Interactive advertising design breaks the traditional way of displaying works at specific locations and times, so that anyone can participate in the interaction of works at different times or places. Before consumers use the product, the first thing that comes into contact with the product is the packaging of the product. Therefore, the level of packaging design directly affects the status and image of the product in the minds of consumers. Interactive packaging that influences consumer behavior can start with people's senses and use a multi-sensory sense of smell, touch, and vision to bombard consumers with a full range of stereoscopic information in order to enhance the competition in the sale of goods and improve the consumer-to-consumer relationship. In the interactive packaging design, designers should follow the three principles of usability, ease of use, and pleasantness. Any new packaging design should be people-oriented, put consumers first, and design around users. Instead of the designer sitting in front of the computer take it for granted. Many designers have been working on product packaging design for a long time, but never thought about the feelings of a consumer who bought the product when they saw the outer packaging of the product. Therefore, we must allow consumers to participate in the process of packaging design. After the product packaging is designed, consumers should be evaluated in a timely manner and problems should be corrected. This is an important means to achieve interactive packaging design [5].

\section{Conclusion}

The interaction design brings a variety of feelings and experiences to the user. In all interaction modes, the interaction feeling and the experience interaction method obtained through visual means are the smoothest information and the simplest use process. Therefore, the application of interaction design in the future visual communication design will be more and more extensive, and people will also pay more and more attention to the interactive experience in visual communication design.

\section{References}

[1] Deng Weibin, Li Xue, Chen Yuanyuan. Research on Innovative Methods of Traditional 
Elements in Product Design [J]. Design, 2016, 29(1): 128-129.

[2] Wang Hao. A review of the development of new media art [M]. Shanghai: Tongji University Press, 2009.67-68.

[3] Zhang Kai, Liu Shuyang. Research on the Construction of Interactive Rules Based on Cognitive Load Adjustment [J]. , 2016, 29(1): 57-59.

[4] Xu Xiaoping, Kong Deshi. Exploring the Teaching Mode of "Showing a Good Story" to Display Design [J]. Design, 2016, 29(1): 114-115.

[5] Tian Yun, Wang Hao. The Application of Metaphor in the Design of Product Interface [J]. 2016, 29(1): 76-77 